\title{
Leader-Following Control System Design Based on Back-stepping Control Strategy
}

\author{
Dong-Hun Lee ${ }^{1}$, Tran-Duc Quan ${ }^{1 *}$ and Young-Bok Kim ${ }^{2 *}$ \\ ${ }^{1}$ Department of Mechanical System Engineering, the Graduate School, Pukyong National University \\ Nam-gu, Busan, Korea; iottoi1 @ naver.com \\ ${ }^{2}$ Department of Mechanical System Engineering, Pukyong National University \\ Nam-gu, Busan, Korea; kpjiwoo@pknu.ac.kr \\ * Corresponding Authors : tducquan@gmail.com,kpjiwoo@pknu.ac.kr
}

\begin{abstract}
In this study, a motion control problem for the vessels towed by tugboats or towing ships on the sea is considered. The towed vessels including barge ships are need to have assistance of tugboats. Combining two vessels, some work purposes in the sea or harbor area can be completed. In this study, the authors give newly developed mathematical model and control system strategy. Especially, the system model fully presenting the physical characteristics of two vessels are derived. For controlling the system effectively, it is considered that the towed vessel has no power propulsion system but the rudder is activated to improve the maneuverability. Considering the strong nonlinearities included in the vessel dynamics, the modelled system is presented by nonlinear system without linearization of nonlinear parameters. Thus, the control system for the towed vessel is designed based on the nonlinear control scheme. Exactly, the backstepping control method is applied to its motion control. Also, the PID control method is applied for comparing with the proposed control strategy.
\end{abstract}

Keywords: towed vessel; tugboat; propulsion system; towing vessel; steering performance; rudder; back-stepping control

\section{Introduction}

The development of control and measurement technology has made remarkable results regardless of its application areas and subjects. Especially, applications to the marine field are increasing and more recently extending to the area where autonomous navigation is possible. Although it is already possible in the aeronautical and land sector in the commercialization level, the application of advanced technology is still relatively limited in the marine industry due to poor and hard ocean environment.

In the early 1990s, different control technologies had begun to be applied in ship motion control problems. The main issue was the problem of path control at a low or constant speed. Therefore, complex and intricate research studies have been carried out. For example, multivariate neural controller design methods for automatic berthing of ships using a multilayer feedforward neural network proposed by Yamato et al. [1] and Zhang et al. [2]. One of the most interesting research on ship motion control is the construction of a ship with four azimuth type propellers [3]. This study has been evaluated as the most leading research result and is being used as a dynamic positioning system (DPS) for FPSO (floating production storage and offloading) and drillship, etc.

In the previous research studies, a given goal was to maintain the right path in the wide ocean $[4,5,6]$. In other words, using the main propulsion device and the rudders, the sailing technologies have been developed.

However, more sophisticated ship motion control strategies are necessary when the ship starts approaching to the harbor. To partially overcome the stability problem of the berthing process, several solutions were proposed. In these cases, the ship is moved in parallel to the seawall with only the side thrusters to complete the final step [7].

More advanced studies for guiding ship with four tugboats [8,9], and damper and rope [10,11,12] have been carried out. The objectives of these studies are to improve the stability of the docking operation at the final stage or restricted maneuvering environment.

The various ship motion control methods and techniques described above are implemented by installing the active control devices on the controlled vessel. In other words, it is natural that the motion control system is equipped on the controlled vessel itself. 
On the sea, non-powered ships (such as barges) are frequently used for transporting cargos. For moving large structures from shipyard to other places, the barge-type vessels are used and dragged by tugboats. In some cases, the ships with propulsion system are towed by tugboats under non-powered state as shown in Figure 1.

In the cases abovementioned, the tugboat leads and controls the towed vessels. It is called as the leader-following system. However, it is difficult to make the towed vessels follow the tugboat's moving route because of loss of maneuverability. Moreover, in harsh sea condition, safe maneuvering may be impossible.

About this issue, Fitriadhy et al. [13] gave an interesting result with mathematical model and course stability analysis. The obtained model presents the dynamics of tugboat and non-powered barge under wind attack. The result shows that the stability of leader-following system strongly depends on the wind attack angle and towing rope length.

However, the system stability and dynamic analysis were undertaken using the linearized model. It is evident that some useful strategy such as something active devices is necessary to occupy system stability and maneuverability.

If we insist on the conventional and inactive methods, more stable and better sailing performance could not be preserved. In the result, the severe accidents may occur due to the wave disturbance and complicated maneuvering environment induced by other vessels passing by. Needless to mention, if the towed vessel deviates from the path of the tugboat, collision possibility with other ships may increase.

Considering this fact, in this study, a new method to obtain a solution to above problem is discussed. To facilitate the maneuverability for non-powered vessel, the authors introduce a novel strategy based on the leader-following concept. It is a control system method for the towed and towing vessel system shown in Figure 2. For giving the minimal function to the powerless towed vessel, it is considered that the vessel has the rudder which is controllable.

Practically, the rudder or portable propulsion system can be additionally provided for the non-powered vessels (such as barge ships) and controlled. Furthermore, the ships sailing in the narrow and restricted waters condition can be controlled by remote control system, tugboats and cooperation of various systems (see Figure 1). In this case, if we use the rudders of the non-powered vessel, needless to say, something excellent and sufficiently improved maneuverability can be obtained.

Therefore, the authors consider that an appropriate number of rudders are installed on the towed vessel. That is, the authors try to improve the steering stability and performance of the non-powered towed vessel without controlling of propulsion force. In this study, the dynamics of controlled vessels is described by nonlinear model, and the nonlinear controller is designed without linearization. The simulation results are given to evaluate the control performance with comparison studies.

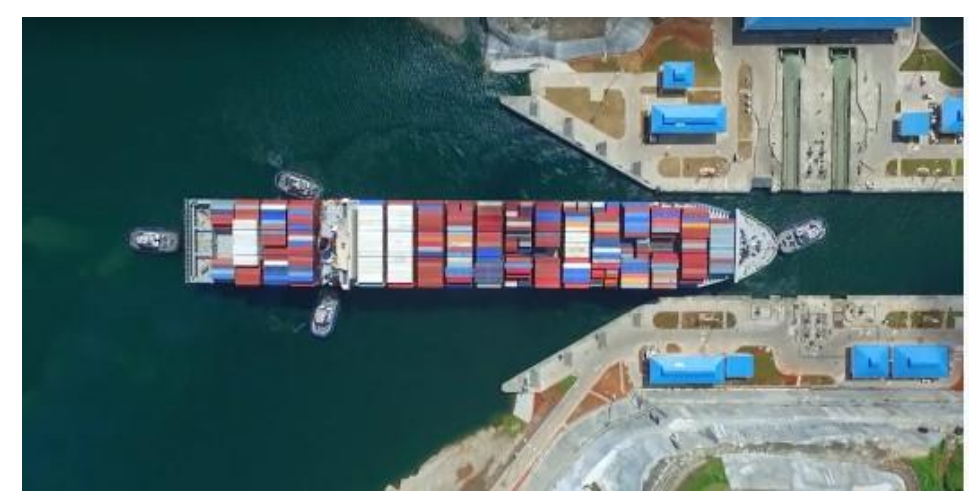

Figure 1. Photo of ship motion control in the hard environment constraints (captured from Panama Canal co.).

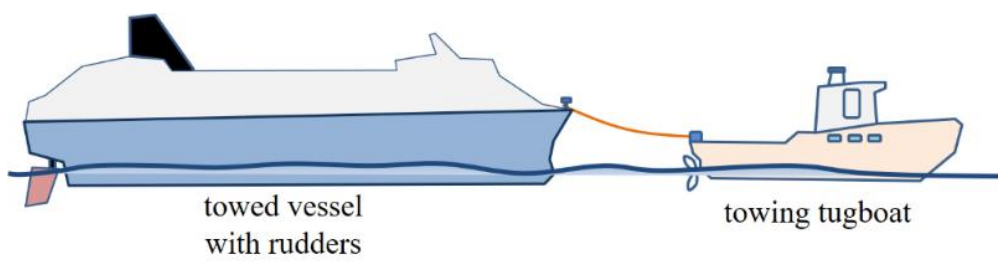

Figure 2. Schematics of the controlled system with active rudders. 


\section{System Modelling and Controller Design}

In order to design the control system, having a mathematical model that describes the dynamics of the system is compulsory. As shown in Figure 3, the towed vessel does not have any self-thrust force based on the assumption given before. Also, it is assumed that two rudders are adequately installed at arbitrary positions to keep robust system stability and improve maneuverability of the towed vessel.

In Figure $3, l_{r}$ is length of rope connecting the towing tugboat and towed vessel. It is assumed that the length change of $l_{r}$ is sufficiently small and the mass of the rope is relatively small.

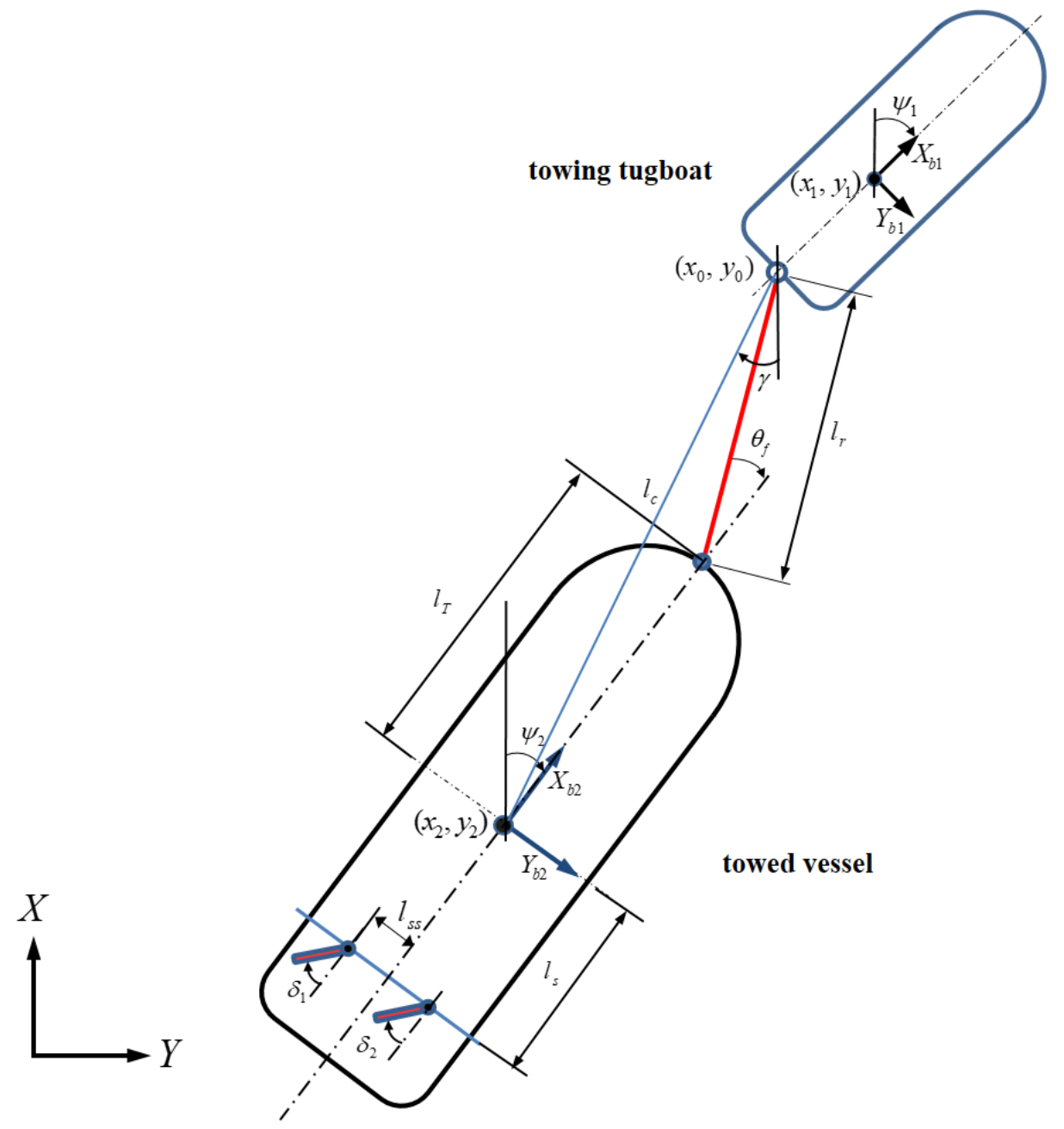

Figure 3. Leader-following system description.

\subsection{Motion Towing Tugboat}

In this study, surge, sway and yaw motions of towing tugboat in the horizontal plane are taken into consideration. Then the 3-DOF equation of motions is derived adequately as follows [3]:

$$
\begin{aligned}
& \dot{\eta}_{1}=R\left(\psi_{1}\right) v_{1} \\
& M_{1} \dot{v}_{1}+D_{1} v_{1}=\tau_{1}-\tau_{\text {rope }}
\end{aligned}
$$

where $M_{1} \in R^{3 \times 3}$ is the inertia matrix; $D_{1}\left(v_{1}\right) \in R^{3 \times 3}$ is the damping matrix which are expressed as follows: 


$$
M_{1}=\left[\begin{array}{ccc}
m_{1}-X_{u 1} & 0 & 0 \\
0 & m_{1}-Y_{\dot{v} 1} & -Y_{r 1} \\
0 & -N_{v 1} & I_{z}-N_{r 1}
\end{array}\right], D_{1}=\left[\begin{array}{ccc}
-X_{u 1} & 0 & 0 \\
0 & -Y_{v 1} & -Y_{r 1} \\
0 & -N_{v 1} & -N_{r 1}
\end{array}\right]
$$

and $\eta_{1}=\left[x_{1}, y_{1}, \psi_{1}\right]^{T} \in R^{3}$ represents position $\left(x_{1}, y_{1}\right)$ and heading angle $\psi_{1}$ in the earth-fixed frame. In addition, $v_{1}=\left[u_{1}, v_{1}, r_{1}\right]^{T} \in R^{3}$ describes the surge, sway and yaw rate of ship motion in the body-fixed frame; $\tau_{1}=\left[\begin{array}{lll}\tau_{x 1} & \tau_{y 1} & \tau_{\psi 1}\end{array}\right]^{T}$ is thrust vector and denotes surge force, sway force and yaw moment around the center of gravity of the towing tugboat. Additionally, the vector $\tau_{r o p e}=\left[\begin{array}{lll}\tau_{x r 1} & \tau_{y r 1} & \tau_{\psi r 1}\end{array}\right]^{T}$ denotes surge force, sway force and yaw moment made by the towing tugboat. Moreover, the rotation matrix $R\left(\psi_{1}\right)$ in Equation (1) in heading direction describes the kinematic equation of motion;

$$
R\left(\psi_{1}\right)=\left[\begin{array}{ccc}
\cos \psi_{1} & -\sin \psi_{1} & 0 \\
\sin \psi_{1} & \cos \psi_{1} & 0 \\
0 & 0 & 1
\end{array}\right]
$$

The parameters shown in Equation3 are summarized as follows:

$m_{1}$ : body mass of towing tugboat

$I_{z 1}:$ moment of inertia about $\mathrm{z}$ axes

$X_{\dot{u}_{1}}, Y_{\dot{v}_{1}}, Y_{\dot{r}_{1}}:$ additional mass

$N_{i 1}, N_{i 1}:$ additional moment of inertia

$u_{1}, v_{1}: x_{1}, y_{1}$ direction velocity component

\subsection{Motion of Towed Vessel}

Same as the description of towing tugboat, the 3-DOF equation of the towed vessel motion is derived adequately as follows:

$$
\begin{aligned}
& \dot{\eta}_{2}=R\left(\psi_{2}\right) v_{2} \\
& M_{2} \dot{v}_{2}+D_{2} v_{2}=\tau_{2}
\end{aligned}
$$

where $\tau_{2}=\left[\begin{array}{lll}\tau_{x 2} & \tau_{y 2} & \tau_{\psi 2}\end{array}\right]^{T}$ denotes surge force, sway force and yaw moment. The actual actuation $\tau_{2}$ of the towed vessel is generated by the traction force of the rope and the rudder angles.

As shown in Figure 3, we consider that the connecting point $\left(x_{0}, y_{0}\right)$ at the tugboat is the tracking target of the towed vessel, since the leading tugboat tows the controlled vessel with rope. It means that the tracking route is continuously made by the rope connecting point of towing vessel. The towed vessel motion can be solved from weather optimal positioning control (WOPC) method [3,14] which is a useful method for deriving a solution on this issue. The parameters shown in Figure 3 are denoted as follows:

$\left(x_{0}, y_{0}\right)$ : connecting point at the towing vessel

$\left(x_{1}, y_{1}\right)$ : gravity center of the towing vessel

$\left(x_{2}, y_{2}\right)$ : gravity center of the towed vessel

$\psi_{2}$ : heading angle of towed vessel

$\gamma:$ relative angle made by the target position and the control position

$\delta_{i}(i=1,2)$ : rudder rotation angle

$l_{r}$ : rope length for connecting two vessels

$l_{T}$ : distance from the gravity center to the rope connecting point of the towed vessel

$l_{s}$ : distance from the center of rudder \#1, \#2 to the gravity center of the towed vessel

$l_{s s}:$ distance from the center of rudder \#1, \#2 to the gravity center of the towed vessel in surge direction. 
The Cartesian coordinates $\left(x_{2}, y_{2}\right)$ is related by using polar coordinates as follows:

$$
\begin{aligned}
& x_{2}=x_{0}+l_{c} \cos \gamma \\
& y_{2}=y_{0}+l_{c} \sin \gamma
\end{aligned}
$$

where,

$$
\begin{aligned}
& l_{c}=\sqrt{\left(x_{2}-x_{0}\right)^{2}+\left(y_{2}-y_{0}\right)^{2}} \\
& \gamma=\operatorname{atan} 2\left(y_{2}-y_{0}, x_{2}-x_{0}\right)
\end{aligned}
$$

The derivative of Equations (7) and (8) are as follows:

$$
\begin{aligned}
& \dot{x}_{2}=\dot{x}_{0}+\dot{l}_{c} \cos \gamma-l_{c} \sin \gamma \dot{\gamma} \\
& \dot{y}_{2}=\dot{y}_{0}+\dot{l}_{c} \sin \gamma+l_{c} \cos \gamma \dot{\gamma}
\end{aligned}
$$

From Equations (11) and (12), $\eta_{2}=\left[x_{2}, y_{2}, \psi_{2}\right]^{T} \in R^{3}$ can be expressed as

$$
\dot{\eta}_{2}=\left[\begin{array}{ccc}
\cos \gamma & -\sin \gamma & 0 \\
\sin \gamma & \cos \gamma & 0 \\
0 & 0 & 1
\end{array}\right]\left[\begin{array}{ccc}
1 & 0 & 0 \\
0 & l_{c} & 0 \\
0 & 0 & 1
\end{array}\right]\left[\begin{array}{c}
\dot{l}_{c} \\
\dot{\gamma} \\
\dot{\psi}_{2}
\end{array}\right]+\left[\begin{array}{cc}
1 & 0 \\
0 & 1 \\
0 & 0
\end{array}\right]\left[\begin{array}{c}
\dot{x}_{0} \\
\dot{y}_{0}
\end{array}\right]
$$

Define the state vectors

$$
p_{0}=\left[\begin{array}{c}
x_{0} \\
y_{0}
\end{array}\right], \mathbf{x}=\left[\begin{array}{c}
l_{c} \\
\gamma \\
\psi_{2}
\end{array}\right]
$$

Then a new kinematic relationship can be written as in terms of the vectors $p_{0}$ and $\mathbf{x}$ :

$$
\dot{\eta}_{2}=R(\gamma) H\left(l_{c}\right) \dot{\mathbf{x}}+L \dot{p}_{0}
$$

where,

$$
R(\gamma)=\left[\begin{array}{ccc}
\cos \gamma & -\sin \gamma & 0 \\
\sin \gamma & \cos \gamma & 0 \\
0 & 0 & 1
\end{array}\right], H\left(l_{c}\right)=\left[\begin{array}{lll}
1 & 0 & 0 \\
0 & l_{c} & 0 \\
0 & 0 & 1
\end{array}\right], L=\left[\begin{array}{ll}
1 & 0 \\
0 & 1 \\
0 & 0
\end{array}\right]
$$

From Equation (15), Equation (5) can be replaced by a differential equation

$$
\dot{\mathbf{x}}=T(\mathbf{x}) v_{2}-T(\mathbf{x}) R^{T}\left(\psi_{2}\right) L \dot{p}_{0}
$$

where $T(\mathbf{x})$ is

$$
T(\mathbf{x})=H^{-1}\left(l_{c}\right) R^{T}(\gamma) R\left(\psi_{2}\right)
$$

To facilitate the convenience of expression, $T(\mathrm{x})$ is defined $T(\mathrm{x})=T, H\left(l_{c}\right)=H, M_{2}(v)=M_{2}, D_{2}(v)=D_{2}$. Therefore, the Equation (17) can be expressed as follows

$$
v_{2}=T^{-1} \dot{\mathbf{x}}+R^{T}\left(\psi_{2}\right) L \dot{p}_{0}
$$

Time differentiation of Equation (17) is as follows:

$$
\dot{v}_{2}=T^{-1} \ddot{\mathbf{x}}+\dot{T}^{-1} \dot{\mathbf{x}}+R^{T}\left(\psi_{2}\right) L \ddot{p}_{0}+\dot{R}^{T}\left(\psi_{2}\right) L \dot{p}_{0}
$$

The towed vessel model Equation (6) is represented by using Equation (17) and substituting Equation (19), (20) such that

where

$$
M_{x} \ddot{\mathbf{x}}+D_{x} \mathbf{x}=T^{-T}\left[q\left(v, \mathbf{x}, \dot{p}_{0}, \dot{p}_{0}\right)+\tau_{2}\right]
$$

$$
\begin{aligned}
& q\left(v_{2}, \mathrm{x}, \dot{p}_{0}, \ddot{p}_{0}\right)=M_{2} R^{T}\left(\psi_{2}\right) L \ddot{p}_{0}+M_{2} \dot{R}^{T}\left(\psi_{2}\right) L \dot{p}_{0}+D_{2} R^{T}\left(\psi_{2}\right) L \dot{p}_{0} \\
& M_{x}=T^{-T} M_{2} T^{-1}, D_{x}=T^{-T} D_{2} T^{-1}
\end{aligned}
$$




\section{Control System Design}

The control goal of the leader-following motion control system is to achieve desirable tracking performance with stability. The positions and heading angles of both towing tugboat and towed vessel will follow desired trajectory in the designed manner. To do this, the towing tugboat is controlled such that a desired trajectory for the follower is made.

Secondly, the towed vessel should be controlled to go after the towing tugboat accurately.

Based on these objectives, the authors design two control systems individually.

\subsection{Control System Design for the Towing Tugboat}

The control structure of towing tugboat is shown in Figure 4. The nonlinear PID controller is applied for the control law to achieve track accuracy control.

$$
\tau_{P I D}=K_{P 1}\left(\eta_{1}-\eta_{d 1}\right)+K_{D 1}\left(\dot{\eta}_{1}-\dot{\eta}_{d 1}\right)+K_{I 1} \int_{0}^{t}\left(\eta_{1}-\eta_{d 1}\right) d t
$$

Where $\eta_{1}$ is the actual motion vector, and $\eta_{d 1}$ is the desired motion vector for surge, sway position and yaw angle, respectively.

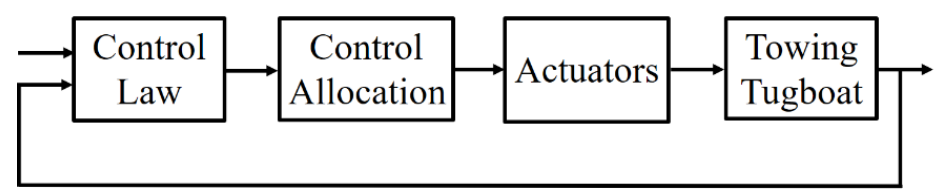

Figure 4. Control structure of towing tugboat with PID control.

\subsection{Control System Design for the Towed Vessel}

The second objective of the leader-following motion control system is controlling the towed vessel to go after the towing tugboat. There are three concepts to obtain this goal. The first requirement is keeping the distance between two vessels to be constant. And, the second requirement is controlling the relative angle made by the target position and the control position towed vessel to be $\gamma_{d}=\psi_{1}+\pi$. And the last requirement is controlling the towed vessel heading angle to be $\psi_{2 d}=\psi_{1}$.

Based on these definitions, a nonlinear control law based on back-stepping technique is designed. Consider the motion of the towed vessel in polar coordinates. We thereafter need to design the control law $\tau_{2}$ such that the system in Equation (23) is stable.

$$
M_{x} \ddot{\mathbf{x}}+D_{x} \dot{\mathbf{x}}=T^{-T} \tau_{2}+T^{-T} q
$$

If we define the control parameter vector as $\mathrm{x}_{d}=\left[\begin{array}{lll}l_{c d} & \gamma_{d} & \psi_{2 d}\end{array}\right]^{T} \in R^{3}$, the control design process based on nonlinear control framework is summarized as follows.

Step 1: Define the error variables

$$
\begin{aligned}
& z_{1}=\mathbf{x}-\mathbf{x}_{d} \\
& z_{2}=\dot{\mathbf{x}}-\alpha
\end{aligned}
$$

Where $z_{1}$ is the tracking error, $\alpha$ is the stabilizing control:

$$
\alpha=\dot{\mathbf{x}}_{d}-\Lambda z_{1}
$$

And $\Lambda>0$ is a diagonal matrix.

From Equations (25) and (26), the derivative of $z_{1}$ can be represented as follows:

$$
\dot{z}_{1}=\dot{\mathbf{x}}-\dot{\mathbf{x}}_{d}=z_{2}-\Lambda z_{1}
$$

Consider a Lyapunov function candidate and its derivative given as Equation (28) and Equation (29), respectively.

$$
\begin{aligned}
& V_{1}=\frac{1}{2} z_{1}^{T} K_{P 2} z_{1} \\
& \dot{V}_{1}=z_{1}^{T} K_{P 2} \dot{z}_{1}=-z_{1}^{T} K_{P 2} \Lambda z_{1}+z_{1}^{T} K_{P 2} z_{2}
\end{aligned}
$$

Where $K_{P 2}>0$ is a diagonal matrix. 
Step 2: From Equation (26), the following expressions are obtained.

$$
\begin{aligned}
& \dot{\mathbf{x}}=z_{2}+\alpha \\
& \ddot{\mathbf{x}}=\dot{z}_{2}+\dot{\alpha}
\end{aligned}
$$

This implies that the towed vessel motion described in Equation (23) can be expressed in terms of $z_{2}, \alpha$ as following.

$$
M_{x} \dot{z}_{2}+D_{x} z_{2}=T^{-T} \tau_{2}+T^{-T} q-M_{x} \dot{\alpha}-D_{x} \alpha
$$

Consider, an additional Lyapunov function candidate is chosen

$$
V_{2}=V_{1}+\frac{1}{2} z_{2}^{T} M_{x} z_{2}
$$

then its time derivative yields

$$
\dot{V}_{2}=\dot{V}_{1}+z_{2}^{T} M_{x} \dot{z}_{2}
$$

Also, the time derivative of $V_{2}$ can be expressed by substitution Equation (29) and (32) to Equation (34) as

$$
\dot{V}_{2}=-z_{1}^{T} K_{P 2} \Lambda z_{1}+z_{1}^{T} K_{P 2} z_{21}+z_{2}^{T}\left[T^{-T} \tau_{2}+T^{-T} q-M_{x} \dot{\alpha}-D_{x} \alpha-D_{x} z_{2}\right]
$$

To satisfy condition $\dot{V}_{2} \leq 0$, the control law should be chosen as

$$
T^{-T} \tau_{2}=-T^{-T} q+M_{x} \dot{\alpha}+D_{x} \dot{\mathbf{x}}-K_{P 2} z_{1}-K_{D 2} z_{2}
$$

where $K_{D 2}>0$ is a diagonal matrix. Then, Equation (35) can be expressed by using Equation (36) as follows:

$$
\dot{V}_{2}=-z_{1}^{T} K_{P 2} \Lambda z_{1}-z_{2}^{T} K_{D 2} z_{2} \leq 0
$$

By Barbalat's lemma, $z_{1}$ and $z_{2}$ approach to zero with $t \rightarrow \infty$.

Finally, the designed control vector is obtained as

$$
\tau_{2}=-q+T^{T}\left[M_{x} \dot{\alpha}+D_{x} \dot{\mathbf{x}}-K_{P 2} z_{1}-K_{D 2} z_{2}\right]
$$

The configuration of the proposed back-stepping control system for the towed vessel can be illustrated as Figure 5.

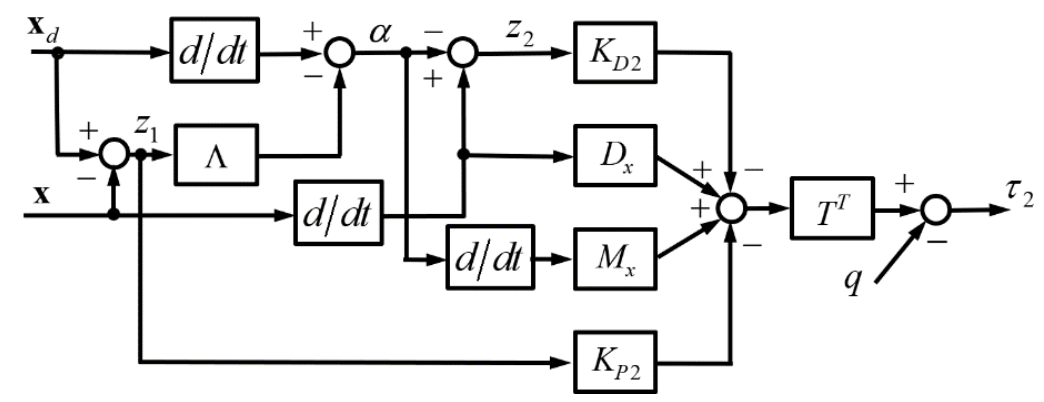

Figure 5. Configuration of the proposed back-stepping controller for the towed vessel.

We need to calculate control gains $\Lambda, K_{P 2}, K_{D 2}$ such that $\dot{V}_{2}$ satisfies the condition $\dot{V}_{2} \leq 0$. Based on the Lyapunov stability theory, it is clear that the system Equation (32) is globally asymptotically stable with the control law of Equation (38). Then, it is preserved that the tracking error and its derivative converges to zero in a finite amount of time.

\section{Simulation Results}

The main objective of the simulation study is to investigate the tracking performance of the towed vessel motion. As abovementioned, the tracking route of the towed vessel is produced by the towing tugboat with PID control. Therefore, the towing tugboat follows the target trajectory and generates the tracking route of towed vessel.

The towed vessel is a model ship which was employed in previous study [9]. For the convenience, it is considered that the towed vessel model is used for towing tugboat also. It means that two vessels are same one.

Subsequently, the parameters of ships represented in Equations (1), (2), (5) and (6) are given as follows: 


$$
\begin{aligned}
& M_{1}=M_{2}=\left[\begin{array}{ccc}
22.5[\mathrm{~kg}] & 0 & 0 \\
0 & 41.5[\mathrm{~kg}] & 0.65[\mathrm{~kg} \cdot \mathrm{m}] \\
0 & 0.65[\mathrm{~kg} \cdot \mathrm{m}] & 5.26\left[\mathrm{~kg} \cdot \mathrm{m}^{2}\right]
\end{array}\right] \\
& D_{1}=D_{2}=\left[\begin{array}{ccc}
1.74[\mathrm{~kg} / \mathrm{s}] & 0 & 0 \\
0 & 6.7[\mathrm{~kg} / \mathrm{s}] & 0.5[\mathrm{~kg} \cdot \mathrm{m} / \mathrm{s}] \\
0 & 0.5[\mathrm{~kg} \cdot \mathrm{m} / \mathrm{s}] & 1.78\left[\mathrm{~kg} \cdot \mathrm{m}^{2} / \mathrm{s}\right]
\end{array}\right]
\end{aligned}
$$

We define the rope length and rudder positions illustrated in Figure 3 as:

$l_{r}=1.0[\mathrm{~m}], l_{T}=1.0[\mathrm{~m}], l_{b}=0.8[\mathrm{~m}], l_{s}=0.7[\mathrm{~m}], l_{s s}=0.1[\mathrm{~m}]$

Parameters of the PID controller for the towing tugboat are chosen as

$$
\begin{aligned}
& K_{P 1}=\operatorname{diag}\{1.4230,3.4992,3.4117\} \\
& K_{D 1}=\operatorname{diag}\{0.2928, \quad 0.7200, \quad 0.7020\} \\
& K_{I 1}=\operatorname{diag}\{0.0018, \quad 0.0044, \quad 0.0043\}
\end{aligned}
$$

The gains of back-stepping controller for controlling the towed vessel are calculated as

$$
\begin{aligned}
& K_{P 2}=\operatorname{diag}\left\{\begin{array}{lll}
4.14, & 4.6, & 3.6
\end{array}\right\} \\
& K_{D 2}=\operatorname{diag}\left\{\begin{array}{lll}
0.07, & 0.18, & 0.4
\end{array}\right\} \\
& \Lambda=\operatorname{diag}\left\{\begin{array}{lll}
0.049, & 0.50, & 0.93
\end{array}\right\}
\end{aligned}
$$

To verify the effectiveness of the proposed control method, its performance is compared with performance of PID control.

The tugboat and the vessel should track the turning reference trajectory with small error as possible as they can. The turning reference is a circle with radius $25[\mathrm{~m}]$ and produced by following equations:

$$
\begin{aligned}
& x_{d}=25 \sin (\pi t / 90) \\
& \left.y_{d}=-25+25 \cos (\pi t / 90) \text { (if } t \leq 180\right), y_{d}=25+25 \cos (\pi t / 90) \text { (if } t>180 \text { ) }
\end{aligned}
$$

Where, the tugboat starts from the initial position set as $x_{01}=0.0[\mathrm{~m}], y_{01}=0.0[\mathrm{~m}], \psi_{01}=0.0[\mathrm{rad}]$. And the distance between the tugboat and the towed vessel is defined as $l_{c 0}=2.0[\mathrm{~m}], \gamma_{0}=0.0[\mathrm{rad}], \psi_{02}=0.0[\mathrm{rad}]$.

In Figure 6, (a) shows the motions of the towing and towed vessels with PID control for the turning route trajectory. The dashed thin line is the reference trajectory, the thick dashed-dot line is the moving route and the heading angle of the towing vessel, whereas the solid line indicates the motion of the towed vessel. Where (b) (d) show the surge, sway motions and yaw angles of two vessels, respectively.

Clearly, the results are not so satisfactory, such that a new control strategy would be provided to improve the control performance.

Then a back-stepping control is applied to the towed vessel control in the same manner of PID control. However, the towing tugboat is controlled by PID control scheme.

The simulation was performed as the process above and results were derived as following.

Figure 7 (a) shows the tracking performance for the turning route as target trajectory. And, (c) (d) illustrate the surge and sway motions and yaw angles two vessels, respectively.

By comparing two results shown in Figure 6 and Figure 7, it is evident that the proposed control scheme works more satisfactorily than the PID control, and an excellent control performance is obtained.

Figure 8 shows the relative motions of towing tugboat and towed vessel. The relative distances are shown in Figure 8 (a), while Figure 8 (b) shows the relative angles. The dashed line is the references whereas the solid line indicates controlled responses, respectively. In additional, the error dynamics $z_{1}$ and $z_{2}$ defined in Equations (24) and (25) are shown in Figure 9. Where the errors are minor and neighborhood around zero. From the Figures 7 9, the towed vessel can always follow the towing tugboat stably and adequately.

In this simulation, only the case where control is activated is introduced. However, it is possible to guess what results are obtained when the control is not performed. And these results can also be confirmed through reference [13].

In the end, it was confirmed through this study that more stable and superior sailing performance can be secured if the rudder function is alive with minimal control function on the towed vessels. 


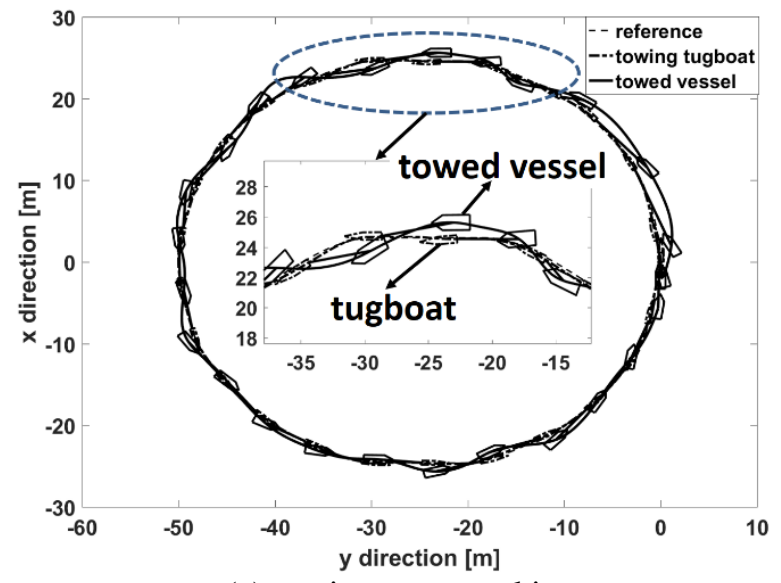

(a) turning route tracking

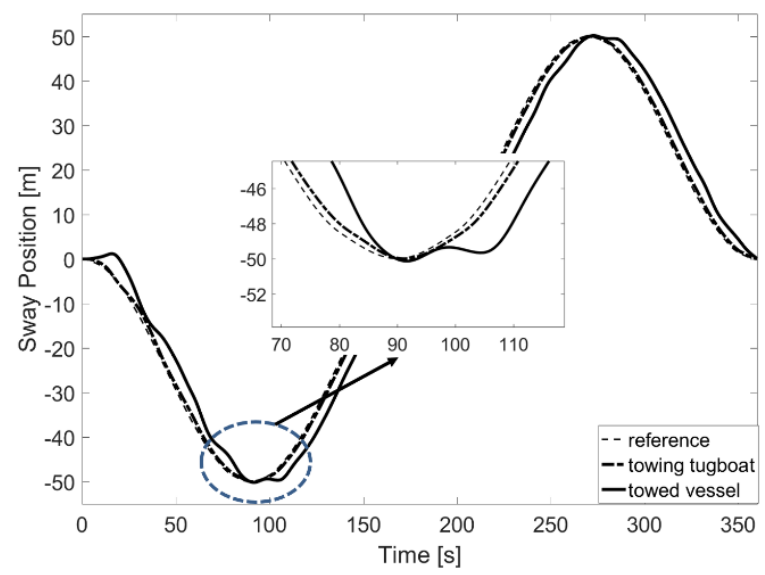

(c) sway motion

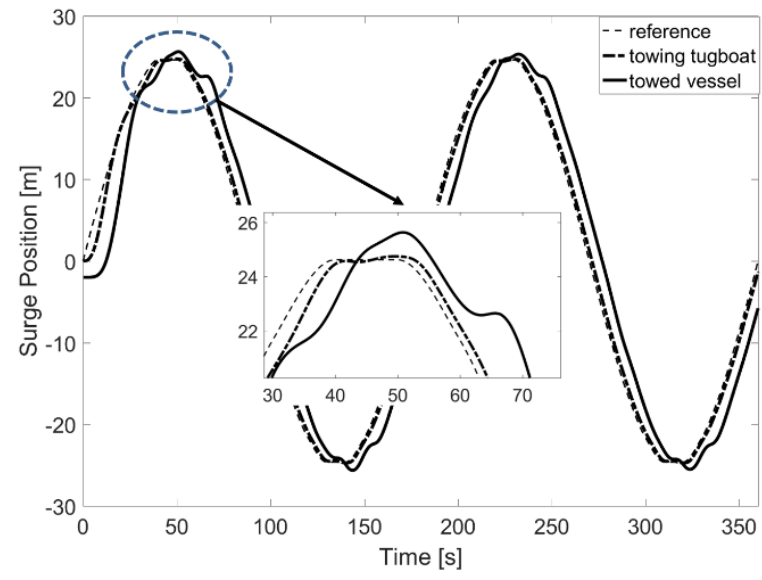

(b) surge motion

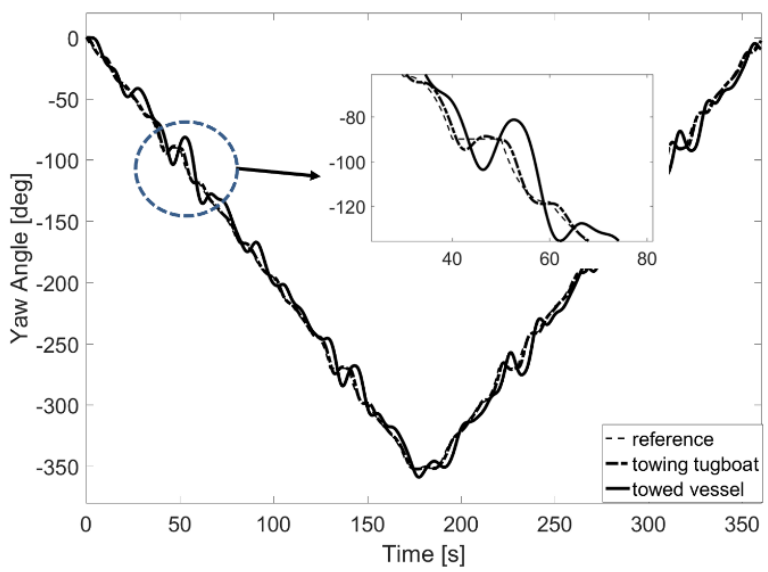

(d) yaw angle

Figure 6. Simulation results with PID control.

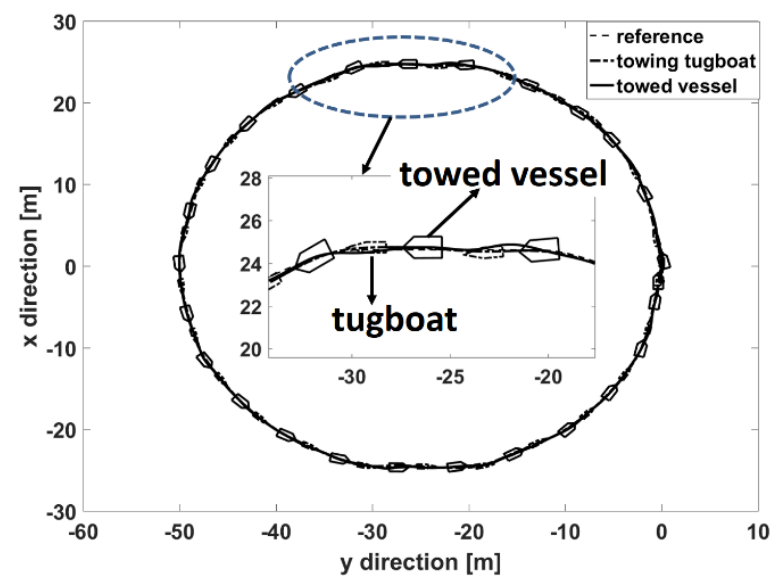

(a) turning route tracking

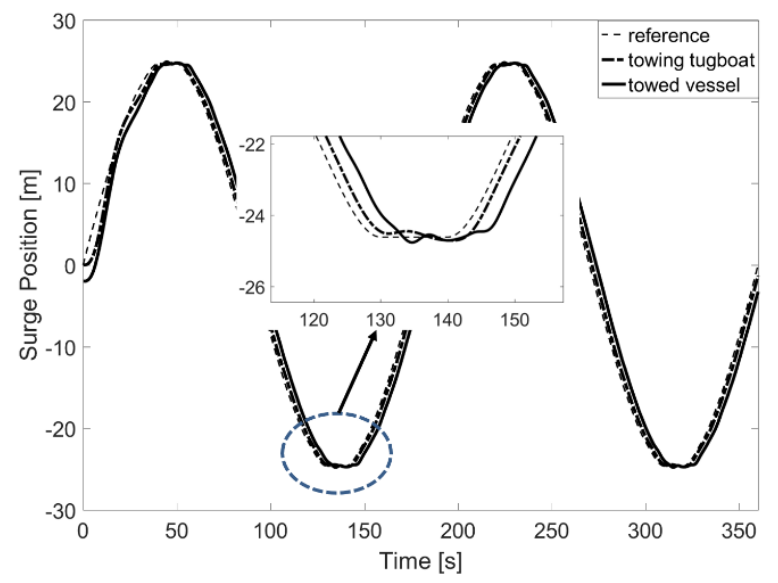

(b) distance 


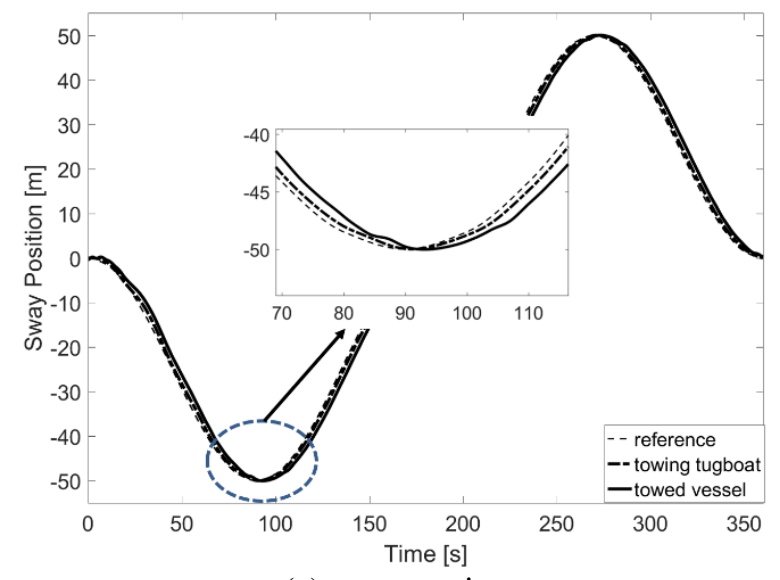

(c) sway motion

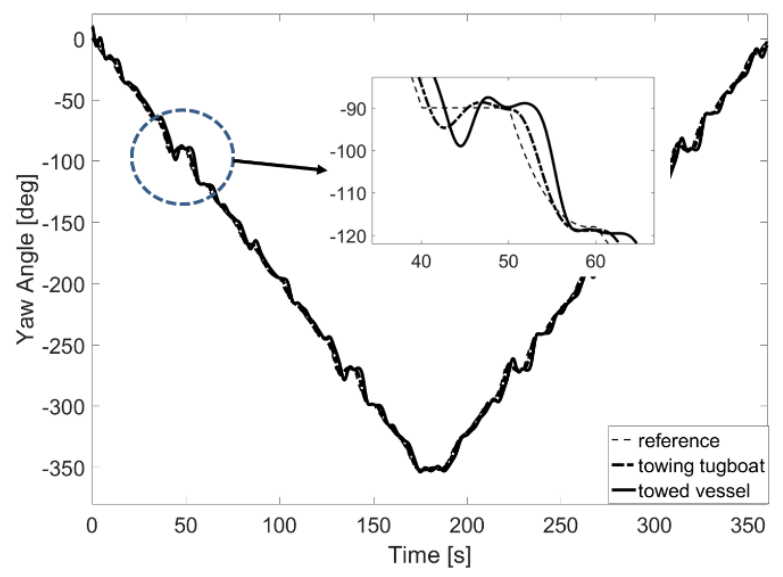

(d) yaw angle

Figure 7. Simulation results with back-stepping control.

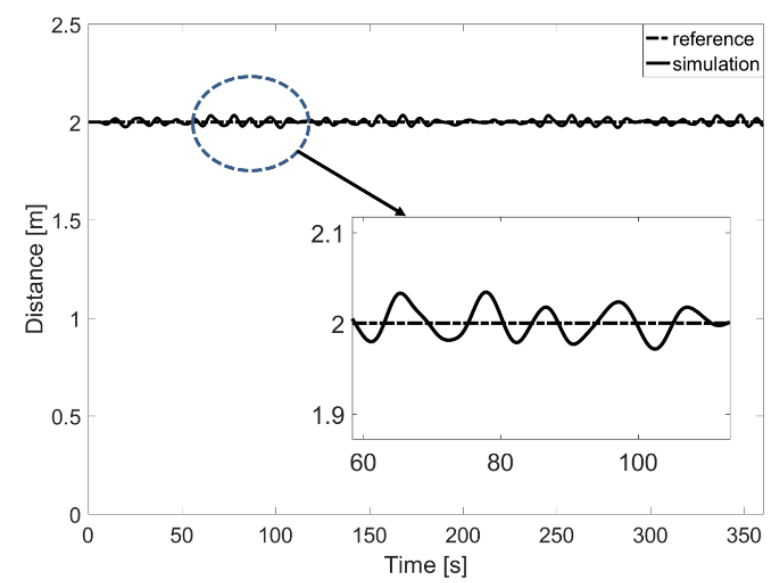

(a) error in distance

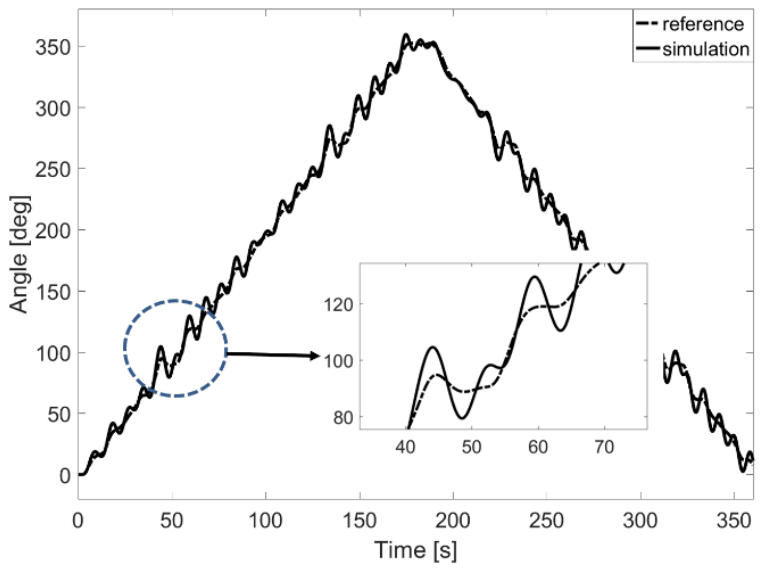

(b) error in angle

Figure 8. Relative errors between towing tugboat and towed vessel.
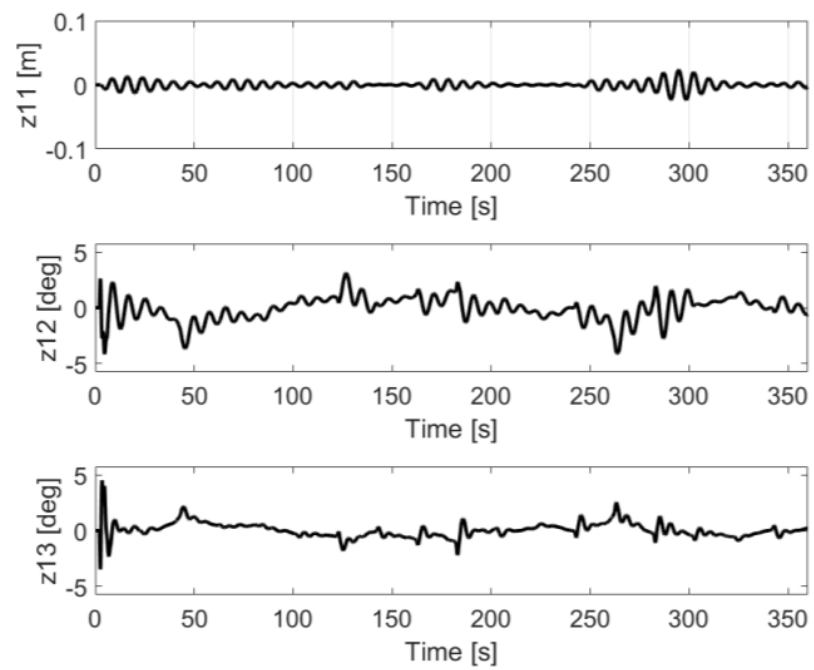

(a) errors in distance and degree $\left(z_{11}=l_{c}-l_{c d}, \mathrm{z}_{12}=\gamma-\gamma_{d}, \mathrm{z}_{13}=\psi_{2}-\psi_{2 d}\right)$ 

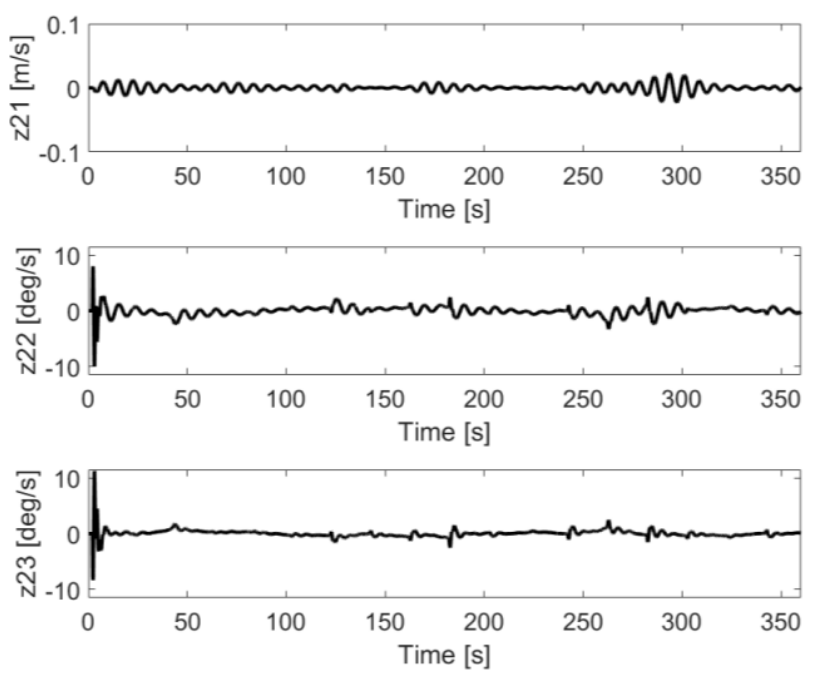

(b) errors in velocity and angular velocity $\left(z_{21}=\dot{l}_{c}-\dot{l}_{c d}, \mathrm{z}_{22}=\dot{\gamma}-\dot{\gamma}_{d}, \mathrm{z}_{23}=\dot{\psi}_{2}-\dot{\psi}_{2 d}\right)$

Figure 9. Error dynamics defined by Equations (24) and (25).

\section{Conclusion}

In this study, the authors have considered the control problem of the lead-following system consisting of two vessels, and proposed a new method to solve this critical issue. The vessels are connected each other with rope and work as the leader and follower, respectively. The towing vessel has the power propulsion system. On the other hand, the towed vessel called as follower is connected to the leader and does not contain any active system except the rudder. Therefore, the safety in sailing and robust maneuverability are not preserved. When it is strongly exposed to hard environment condition, a serious collision accident may not be unavoidable. To overcome this limitation, the authors proposed a new control strategy by activating the rudders in the towed vessel. In this system configuration, the rudders are newly installed on the towed vessel or used as it has, such that the towed vessel can occupy robust motion control ability at last.

The full mathematical model of two connected vessels has been newly obtained, and a nonlinear back-stepping controller are applied for simulation study. The simulation results with comparison study demonstrate that the proposed control strategy preserve the accurate tracking performance and safe sailing of towed vessel.

The proposed method is applicable to the control of various marine systems in a variety of settings.

\section{References}

1. Yamato, H.; Uetsuki, H.; Koyama, T. Automatic berthing by the neural controller. Proc. 9th Ship Control Systems Symposium USA 1990, 3, 183-201.

2. Zhang, Y.; Hearn, G.E.; Sen, P. A multivariable neural controller for automatic ship berthing. Journal of IEEE Control System 1999, 17, 31-44.

3. Fossen, T.I.; Marine Control System-Guidance, Navigation, Rigs and Underwater Vehicle, Trondheim, Norway: Norwegian University of Science and Technology 2002.

4. Lopez, M.J.; Rubio, F.R.; LQG/LTR control of ship steering autopilots. Proceedings of the 1992 IEEE International Symposium on Intelligent Control 1992, 447-450.

5. Michiel, W.; Erjen, L.; Kristin, Y.P.; Henk, N. Output feedback tracking of ships. IEEE Transactions on Control Systems Technology 2011, 19, 2, 442-448.

6. Zwierzewicz, Z. On the ship trajectory tracking LQG controller design. Transactions on the Built Environment 1999, 42, 399-406.

7. Bui, V.P.; Jeong, J.S.; Kim, Y.B.; Kim, D.W. Optimal control design for automatic ship berthing by using bow and stern thrusters. Journal of Ocean Engineering and Technology 2010, 24, 2, 10-17.

8. Bui, V.P.; Kawai, H.; Kim, Y.B.; Lee, K.S. A ship berthing system design with four tug boats. Journal of Mechanical Science and Technology 2011, 25, 5, 1257-1264. 
9. Bui, V.P.; Kim, Y.B. Development of constrained control allocation for ship berthing by using autonomous tugboats. International Journal of Control Automation and Systems 2011, 9, 6, 1203-1208.

10. Tran, A.M.D.; Ji, S.W.; Kim, Y.B. A ship berthing system design by cooperating with tugboats and dampers. Journal of Drive and Control 2014, 11, 3, 7-13.

11. Tran, A.M.D.; Kim, Y.B. Dynamic identification and robust control performance evaluation of towing rope under rope length variation. Journal of the Korean Power System Engineering 2016, 20, 2, 58-65.

12. Lee, D.J.; Kim, J.K.; Kim, B.S. Design of an automatic winch system for small fishing vessel. Journal of the Korean Society for Fisheries and Technology 2000, 36, 3, 157-165.

13. Fitriadhy A.; Yasukawa, H.; Koh, K.K. Course stability of a ship towing system in wind. Ocean Engineering 2013, 64, $135-145$.

14. Tran, D.Q.; Lee, D.H.; Kim T.W., Kim Y.B. Park, H.C. A study on maneuvering performance improvement of a towed vessel without a power propulsion system: system modeling. Journal of the Korean Society of Fisheries and Ocean Technology 2018, 54, 4, 343-352. 